\title{
Limit to Cardiomyocyte Hypertrophy in Right and Left Ventricles: Possible Precursors of Systolic Heart Failure
}

\section{Richard E Tracy*}

Pathologist for Orleans Parish Forensic Center, LA, USA

*Corresponding Author: Tracy RE, Pathologist for Orleans Parish Forensic Center, LA 70125, USA, Tel: 1-504-568-6072; E-mail: rtracy@lsuhsc.edu

Received date: September 15, 2016; Accepted date: October 17, 2016; Published date: October 21, 2016

Copyright: (C) 2016 Tracy RE. This is an open access article distributed under the terms of the Creative Commons Attribution License, which permits unrestricted use, distribution, and reproduction in any medium, provided the original author and source are credited.

\begin{abstract}
Cardiac myocytes respond to volume overload by undergoing hypertrophy in two phases: Proportionate increase in breadth and length and Continuation only of lengthening after the chamber dilates beyond a defined threshold. These responses have upper limits that are in need of explanation. Hearts were retrieved from 104 forensic autopsies. Myocyte breadths were measured in H\&E-stained paraffin sections from LV and RV free walls. Mean myocyte lengths were calculated from chamber diameter and wall thickness (at full systole in these preparations) using a previously defined regression equation. Using body weight as an estimate for volume overload, myocyte breadth obeyed the expected two phase pattern. This pattern differed between ventricles in only one well defined way: the limiting mean breadths in the right and left respectively were $17.5 \mu \mathrm{m}$ and $21.9 \mu \mathrm{m}$. Hence, in the RV it is not the attained size that imposed the limit on further hypertrophy of the mean myocyte because that attained size imposed no such limit in the LV. The behaviour of overworked myocytes is virtually identical in the two ventricles and it seems reasonable to propose that this identity should affect also the upper limit to size. Log normal distributions generally fit reasonably well in both RV and LV with no ceiling exerted upon the most enlarged cells, which further supports the lack of effect by attained size. A body of theory emerges which provisionally places a causal role for hypertrophy on the simple operation of geometry constrained by a fixed number of myocytes. The theory emerged without need to introduce any proposed contractile weakening. If this theory is confirmed by further observations using improved methodology then this conclusion would sharply constrain what subcellular mechanisms might mediate these events.
\end{abstract}

Keywords: Aging; Human; Hypertension; Obesity

Abbreviations: LV: Left Ventricle; RV: Right Ventricle; LVDs: LV systolic chamber diameter in the high chordal plane $(\mathrm{mm})$; s: Systolic; d: Diastolic; MyR: RV mean myocyte cross sectional breadth $(\mu \mathrm{m})$; MyL: LV mean myocyte cross sectional breadth $(\mu \mathrm{m})$; MFS: LV midwall fractional shortening during a heartbeat (\%); SV: Stroke Volume (ml); Rs: Systolic chamber radius ( $\mathrm{mm})$; Rd: Diastolic chamber radius $(\mathrm{mm})$.

\section{Introduction}

The size of the left ventricle (LV) tends to increase progressively in response to pressure overload and volume overload. It is widely recognized that pressure and volume overloads initially act to expand the $\mathrm{LV}$ myocyte transverse dimension ( $\mathrm{MyL}=$ breadth of $\mathrm{LV}$ myocytes) and that volume overload enhances myocyte length throughout to range of hypertrophy [1-6].

These forms of hypertrophy confront ceilings imposed upon cellular breadths and lengths by mechanisms that are not clearly understood. Considering hypertrophy of cell breadth, it is often proposed that the largest myocytes cease to grow, or perhaps fall away by necrosis or apoptosis, or perhaps undergo longitudinal splitting [7], after reaching a barrier to what is biologically possible.

Such proposals all agree that the limit must be imposed upon each individual cell as it reaches a critical attained size. The data reported here raise reasons for caution in approaching these proposals.
In previous reports from this laboratory concerning the $\mathrm{LV}$ it was found that the frequency distribution curves for MyL in a series of 104 specimens closely followed the log normal form with a trend for the coefficient of variation to remain constant throughout the processes of hypertrophy $[8,9]$, thereby displaying allometric growth patterns $[10,11]$.

That outcome would require that myocytes of all sizes must be restrained alike so that the frequency distribution retains its shape throughout the process $[4,8]$. Limits imposed only upon the largest cells should truncate the upper tail of the bell shaped curve, a finding not seen in the LV data.

This report extends the previous approach to include the right ventricle (RV). The models that are explored here begin by emphasizing the LV chamber diameter in systole (LVDs), which is the condition of the LV at autopsy, and then computing chamber dimensions using spherical and cylindrical models $[12,13]$.

A similar specification is overly complex in the RV because of its departure from any simple shape such as circular or elliptic cylinder. It was elected here, therefore, to refer the RV measurements to the LVDs as done for the LV, thereby utilizing LVDs consistently as the standard of reference for both LV and RV. A logical basis for this decision is considered in the discussion.

The kinds of data examined here are accessible only at autopsy. Forensic pathologists are the front line observers who are the only ones in position to pursue these matters in full. It would be of some value if this report can promote ongoing discussions. 


\section{Methods}

\section{Subjects}

During accessible days between November 2007 and September 2012, the Orleans Parish Coroner's Office supplied 104 specimens. All cases with cardiomegaly (whole heart weight $>399 \mathrm{~g}$ in women and $>449 \mathrm{~g}$ in men) were retained, other than those with right ventricular, valvular, or ischemic disorders. Non-cardiovascular cases without cardiomegaly were chosen to have $<12 \mathrm{~h}$ post mortem intervals, no immediately preceding hospitalization, and cause of death unrelated to cardiovascular disease. The Institutional Review Board declared this autopsy study exempt from their review.

\section{Preparations for histology}

A slice of myocardium presenting the high chordal plane was formalin fixed for 1 to 3 weeks. Duplicate samples were excised perpendicular to this plane from lateral and posterior LV walls and from the RV free wall. Paraffin-embedded samples were sectioned at 6 $\mu \mathrm{m}$ and stained with $\mathrm{H} \& \mathrm{E}$. To adjust for shrinkage in paraffin sections, a unit conversion factor of 1.21 was applied to subsequent histological measurements [8], although this is not needed for the relative comparisons of myocyte breadths in specimens reported here.

\section{Myocyte cross sectional profiles, $\mathrm{MyR}$ and $\mathrm{MyL}$}

These profiles were evaluated by imposing an ellipse upon each image and measuring the minor axis of the idealized outline, calling this the myocyte breadth (MyR and MyL for averages in the right and left). These measurements were made upon black-and-white photographic prints using a digital caliper accurate to $0.01 \mathrm{~mm}$. Measurements were taken in 60 anucleate profiles from sites showing the best cross sectional profiles (4 LV sites and $2 \mathrm{RV}$ sites).
Left ventricular hypertrophy (LVH): LVH was considered present if $\mathrm{LVW}>219 \mathrm{~g}$ in men or $>199 \mathrm{~g}$ in women. The distinction of LVH present or absent is used in one graph.

Midwall fractional shortening (MFS): A previously determined relationship between chamber dilatation and MFS [5] was found to be of relevance to the topics of this report. Those findings are adapted here for the current discussion. They are derived from published echocardiographic data on ventricular dimensions during the cardiac cycle.

\section{Statistical methods}

These are all commonly used techniques available in the SAS programs (SAS Institute, Cary NC, 27512-8000). The frequency distributions for the base -10 logarithms of MyR and MyL were examined for normality using the Shapiro-Wilk test for simplicity and consistency with past reports in this series. The skewness coefficient is the third order moment around the mean.

\section{Results}

Log normal fit to an example of $\mathrm{MyR}$ at the limit to hypertrophy: Figure 1 plots the fitting of a log normal curve to a data pool formed by merging the three specimens with the largest myocyte breadths, i.e., most transversely hypertrophied, in this series of 104 cases. The log normal fit is not rejected by the Shapiro -Wilk test of normality $(p=0.17)$. Coefficients for skewness and kurtosis did not reject normality in this pool of three cases $(-0.095$ and -0.15 respectively, $\mathrm{N}=60 \times 2 \times 3=$ myocytes $\times$ sites $\times$ cases $=360$ myocytes).

Log normal fits in all 104 specimens, MyR: The significance level used to declare misfit to the log normal by the Shapiro-Wilk test was taken to be $\mathrm{p}<0.1$, and this happened in 19 of the 104 specimens (Table $1)$.

\begin{tabular}{|c|c|c|c|c|c|c|}
\hline \multirow[b]{3}{*}{ P-level ${ }^{*}$} & \multicolumn{3}{|c|}{ Left ventricle } & \multicolumn{3}{|c|}{ Right ventricle } \\
\hline & \multirow{2}{*}{$\begin{array}{l}\mathrm{N} \text { O/E† } \\
\text { All }\end{array}$} & \multicolumn{2}{|c|}{ Coefficient of skewness $\ddagger$} & \multirow{2}{*}{$\begin{array}{l}\mathrm{N} \text { O/E† } \\
\text { All }\end{array}$} & \multicolumn{2}{|c|}{ Coefficient of skewness§ } \\
\hline & & $>-0.140$ & $<-0.141$ & & $>-0.050$ & $<-0.051$ \\
\hline$>0.8$ & $8 / 20.8$ & $8 / 11.2$ & $0 / 9.6$ & $12 / 20.8$ & $7 / 9.8$ & $5 / 11.0$ \\
\hline $0.6-0.79$ & $11 / 20.8$ & $10 / 11.2$ & $1 / 9.6$ & $14 / 20.8$ & $8 / / 9.8$ & $6 / 11.0$ \\
\hline $0.4-0.59$ & $16 / 20.8$ & $8 / 11.2$ & $8 / 9.6$ & $17 / 20.8$ & 9/9.8 & $8 / 11.0$ \\
\hline $0.2-0.39$ & $16 / 20.8$ & $11 / 11.2$ & $5 / 9.6$ & $25 / 20.8$ & $11 / 9.8$ & $14 / 11.0$ \\
\hline $0.1-0.19$ & $20 / 10.4$ & $8 / 5.6$ & $12 / 4.8$ & $17 / 10.4$ & $7 / 4.9$ & $10 / 5.5$ \\
\hline $0-0.09$ & $33 / 10.4$ & $11 / 5.6$ & $22 / 4.8$ & $19 / 10.4$ & $7 / 4.9$ & $12 / 5.5$ \\
\hline Chi-square & 2.7 & 8.2 & 285.7 & 18.7 & 3 & 23.2 \\
\hline$P$ & $<0.0001$ & $<0.05$ & $<0.0001$ & $<0.0005$ & $<0.40$ & $<0.0001$ \\
\hline \multicolumn{7}{|c|}{$\begin{array}{l}\text { * Probability by Shapiro-Wilk test that the observed distribution of myocyte breadths fits the log normal form. } \\
\dagger \mathrm{N} O / E=\text { Numbers of specimens Observed over Expected. } \\
\text { † The mean skewness coefficient in these } 104 \text { cases is }-0.140 \text {. } \\
\text { § The mean skewness coefficient in these } 104 \text { cases is }-0.051 \text {. }\end{array}$} \\
\hline
\end{tabular}

Table 1: Number of cases observed/expected according to p-levels of significance tests for fit of LV myocyte breadth to log normal distribution according to skewness of the logarithmically transformed breadths. 
Citation: Tracy RE (2016) Limit to Cardiomyocyte Hypertrophy in Right and Left Ventricles: Possible Precursors of Systolic Heart Failure. J

Page 3 of 7

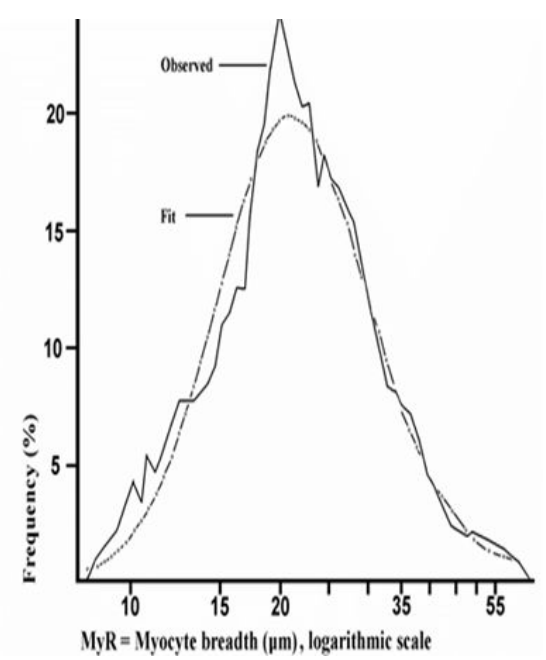

Figure 1: Plots of observed frequencies for RV myocytes versus log transformed myocyte breadth groupings compared with a fit of log normal distribution.

This is an excess of 8.6 more specimens than the expected 10.4 and this 8.6 differs significantly from zero (Chi square $=7.90, \mathrm{df}=1$, $\mathrm{p}<0.0025)$. Hence the log normal is not a rigorously perfect fit for all cases in this series. The results for $\mathrm{LV}$, i.e. MyL, are similar to those in the RV (Table 1).

Relationship of log normal skewness to MyR: In Figure 2, the 19 cases that rejected the log normal fits by the Shapiro-Wilk test are depicted by circles. Denotations of $\mathrm{p}=0.05$ on the vertical axis locate rejection of the normal because of skewness in excess of normal expectations. These complementary significant tests for normality are not always in agreement. The circles that fall below the lower cutoff line for significant skewness $(\mathrm{p}<0.05)$, include most, if not all, of the 8.6 excess Shapiro-Wilk misfits, because circles are scarce in the other two horizontal bands ( 3 in the upper and 5 in the middle band). These 10 cases, i.e. those with significant negative skewness, seem uniformly dispersed across the horizontal, which shows no visible tendency in this lower band of the graph for negative skewness to favor the most hypertrophied specimens. Rather, the instances of excess positive skewness are confined to those with $\mathrm{MyR}<16 \mu \mathrm{m}$.

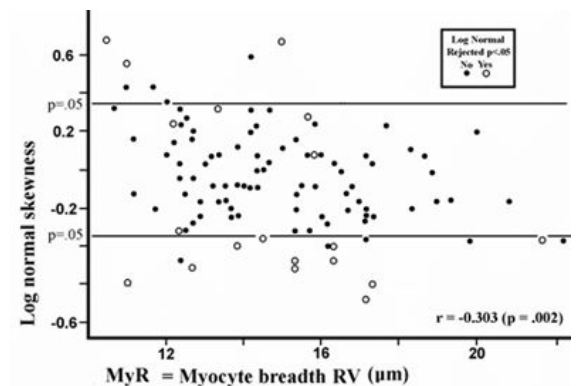

Figure 2: Skewness coefficients for log normal fits versus mean myocyte breadths in 104 specimens. The circles denote poor fits to the log normal at $\mathrm{p}<0.05$ by the Shapiro-Wilk test. The p-levels on the ordinate are those for the cut off level for probability that the skewness coefficients fit the log normal form

The lack of upper tail truncation seen in Figure 1 can therefore be generalized to the dominant patterns in all groupings irrespective of myocyte hypertrophy.

\begin{tabular}{|c|c|c|c|c|c|c|c|}
\hline \multicolumn{4}{|c|}{ Left ventricle† } & \multicolumn{4}{|c|}{ Right ventricle $\dagger$} \\
\hline $\operatorname{MyL}(\mu \mathrm{m})$ & Skewness $\ddagger$ & P-level§ & $\mathrm{N}$ cases & $\operatorname{MyR}(\mu \mathrm{m})$ & Skewness $\ddagger$ & P-level§ & $\mathrm{N}$ cases \\
\hline $12.9-14.9$ & $-0.07 \mathrm{AB}$ & $0.18 \mathrm{~A}$ & 12 & $0.5-11.9$ & $0.20 \mathrm{~B}$ & $0.26 \mathrm{~A}$ & 10 \\
\hline $15.0-16.9$ & $-0.04 \mathrm{~B}$ & $0.19 \mathrm{~A}$ & 15 & $12.0-13.9$ & $-0.06 \mathrm{AB}$ & $0.37 \mathrm{~A}$ & 33 \\
\hline $17.0-18.9$ & $-0.13 A B$ & $0.31 \mathrm{~A}$ & 26 & $14.0-15.9$ & $0.02 A B$ & $0.42 \mathrm{~A}$ & 29 \\
\hline $19.0-20.9$ & $-0.20 \mathrm{~A}$ & $0.28 \mathrm{~A}$ & 25 & $16.0-17.9$ & $-0.22 \mathrm{~A}$ & $0.35 \mathrm{~A}$ & 21 \\
\hline $21.0-22.9$ & $-0.18 A B$ & $0.26 \mathrm{~A}$ & 16 & $18.0-19.9$ & $-0.10 A B$ & $0.51 \mathrm{~A}$ & 7 \\
\hline $23.0-26.5$ & $-0.20 \mathrm{AB}$ & $0.22 \mathrm{~A}$ & 10 & $20.0-22.2$ & $-0.18 A B$ & $0.28 \mathrm{~A}$ & 4 \\
\hline \multicolumn{8}{|l|}{ ANOVA } \\
\hline $\mathbf{F}$ & 1.75 & 1.23 & & & 4.18 & 0.98 & \\
\hline $\mathbf{P}$ & 0.13 & 0.3 & & & 0 & 0.43 & \\
\hline \multicolumn{8}{|c|}{$\begin{array}{l}\text { * Means within a column that fail to share a symbol A or B differ significantly at } p<0.05 \text { by Tukey multiple rangetest. } \\
\dagger \text { Weighted means }(\mathrm{SD}) \text { are } 18.9(0.66) \mu \mathrm{m} \text { for MyL and } 14.8(0.58) \mu \mathrm{m} \text { for MyR. } \\
\text { † Skewness coefficient i.e. third order moment around the mean. } \\
\text { § Probability for Shapiro-Wilk } p \text {-level testing the fit of observed distribution of myocyte breadths to the log normal form. Between P-level and Skewness } r=0.13(p= \\
0.21) \text { for RV and } r=0.36(p=0.00) \text { for LV. }\end{array}$} \\
\hline
\end{tabular}

Table 2: Skewness and P-level for rejection of the log normal fits according to mean myocyte breadth in right and left ventricles. 
Relationship of MyR to rejections of log normal and to negative skewness: Table 2 documents the lack of correlation between MyR and the two quantities, skewness of the log normal fit and p-level for Shapiro-Wilk rejection of the log normal shape. This p-level did not vary significantly across $\mathrm{MyR}$ groupings (mean $\mathrm{p}$-level ranges from 0.26 to 0.51 among the groups). Also, the skewness coefficient did not correlate with MyR when MyR exceeded $11.9 \mu \mathrm{m}$.

Although the excessive misfitting of the log normal curves and the negative skewness both happen too often in the 104 case data set, nevertheless those events happen equally often at all sizes of $\mathrm{MyR}>11.9$ $\mu \mathrm{m}$, and therefore occur without regard to hypertrophy of myocyte breadth. Table 2 also reproduces comparable findings for MyL, i.e. in the left ventricle.

Correlation of mean $\log (\mathrm{MyR})$ to p-level for skewness: The correlation is $\mathrm{r}=-0.27$ ( $\mathrm{p}=0.00$ for falsely rejecting zero). The comparable correlation for MyL in the LV is $\mathrm{r}=-0.19(\mathrm{p}=0.05)$ (Table $3)$.

\begin{tabular}{|c|c|c|c|c|c|}
\hline \multirow[b]{3}{*}{ Variable } & \multirow[b]{3}{*}{ Mean } & \multirow[b]{3}{*}{ SD } & \multicolumn{3}{|c|}{ Product moment correlation coefficients } \\
\hline & & & RV skewness & LV skewness & LVDs \\
\hline & & & $r(p)$ & $r(p)$ & $r(p)$ \\
\hline \multicolumn{6}{|l|}{$\log (M y R)$} \\
\hline Mean & 1.133 & 0.07 & $0.27(0.00)$ & $-0.09(0.35)$ & $0.54(0.00)$ \\
\hline $\mathrm{CV}$ & 11.9 & 1.55 & $0.09(0.34)$ & $0.02(0.84)$ & $-0.15(0.13)$ \\
\hline Skewness & -0.051 & 0.284 & . & $0.03(0.76)$ & $-0.15(0.12)$ \\
\hline Kurtosis & -0.071 & 0.512 & $-0.04(0.68)$ & $-0.20(0.04)$ & $-0.11(0.25)$ \\
\hline P-level ${ }^{*}$ & 0.377 & 0.28 & $0.13(0.21)$ & $0.07(0.46)$ & $0.03(0.75)$ \\
\hline \multicolumn{6}{|l|}{$\log (\mathrm{MyL})$} \\
\hline Mean & 1.235 & 0.071 & $-0.14(0.03)$ & $-0.19(0.05)$ & $0.60(0.00)$ \\
\hline $\mathrm{CV}$ & 11.9 & 1.2 & $0.03(0.73)$ & $-0.09(0.36)$ & $-0.05(0.63)$ \\
\hline Skewness & -0.143 & 0.212 & $0.03(0.76)$ & - & $-0.76(0.64)$ \\
\hline Kurtosis & -0.139 & 0.286 & $0.12(0.23)$ & $-0.35(0.00)$ & $-0.14(0.15)$ \\
\hline P-level ${ }^{*}$ & 0.28 & 0.27 & $0.02(0.81)$ & $0.37(0.00)$ & $0.09(0.37)$ \\
\hline
\end{tabular}

Table 3: Descriptive statistics for selected logarithmically transformed variables.

In the scatter plot of Figure 2, it could be proposed that this significant correlation happens because hypertrophied specimens have an absence of subjects with significant positive skew. This topic calls for a larger number of cases and cannot be resolved here.

Coefficients of variation ( $\mathrm{CV}=\mathrm{SD} / \mathrm{Mean}$ ) in $\mathrm{RV}$ and $\mathrm{LV}$ (data not tabulated): The CV's of the log normal fits did not correlate significantly with the means across the 104 cases ( $r=-0.09$ for RV and -0.03 for $L V, p=0.34$ and 0.73 respectively). These findings meet the requirement for allometric growth.

Variations among replicate samples in RV (data not tabulated): The differences between duplicate samples of the right ventricular free wall departed significantly from zero at $\mathrm{p}<0.05$ by t-tests in 40 of the 104 cases rather than the expected 5.2 cases. By components of variance analysis the variance between sites averaged $7.14 \% \pm 0.83$ SEM of the error variance within sites. This between sites percentage did not correlate significantly across the 104 cases with $\mathrm{MyR}(\mathrm{r}=-0.07, \mathrm{p}=0.44)$, Shapiro-Wilk p-level $(\mathrm{r}=-0.03, \mathrm{p}=0.72)$, or $\log$ normal skewness $(\mathrm{r}=-0.051 \mathrm{p}=0.60)$.
Variations among replicate samples in LV (data not tabulated): With mean MyL measured at $4 \mathrm{LV}$ free wall sites, the means can be compared in 6 pairings for checking differences between pairs $(\mathrm{N}=104$ $\times 6=624$ pairs). The $p$-level for falsely rejecting zero fell at $p<0.05$ in 174 of the $624 \mathrm{t}$-tests rather than the expected 31 , which is $28 \%$ rather than $5 \%$, a clearly significant difference. By components of variance analysis, the percentage of variance between sites averaged $6.28 \% \pm$ 0.10 SEM of the error variance within sites. Across the 104 cases this between sites percentage did not correlate significantly with MyL $(\mathrm{r}=0.02, \mathrm{p}=0.77)$ or Shapiro-Wilk $\mathrm{p}$-level $(\mathrm{r}=-0.03, \mathrm{p}=0.69)$, but did correlate significantly with skewness $(r=0.21, p=0.02)$. This last $r=0.21$ is not a negative coefficient which contradicts the hypothetical inverse relationship.

Myocyte breadth (MyR) versus LV chamber systolic diameter (LVDs): This association is presented in Figure 3 as a scatter plot of the 104 cases. The three uppermost circles locate the three cases that were pooled to construct Figure 1. The sloping solid line is chosen to pass through the graphic origin so as to express constant proportionality. The flat line shows rightward progression of MyR as LVDs dilates beyond $40 \mathrm{~mm}$; this line passes through the mean MyR for the 28 cases 
having LVDs $>40 \mathrm{~mm}$ (MyR mean=17.5 $\mu \mathrm{m}, \mathrm{SD}=2.5 \mu \mathrm{m})$. The dashed line is adapted from a similar plot previously determined for the left ventricle; it depicts the condition with average $\mathrm{MyL}=21.9 \mu \mathrm{m}$ prevailing rightward of the cutoff point.

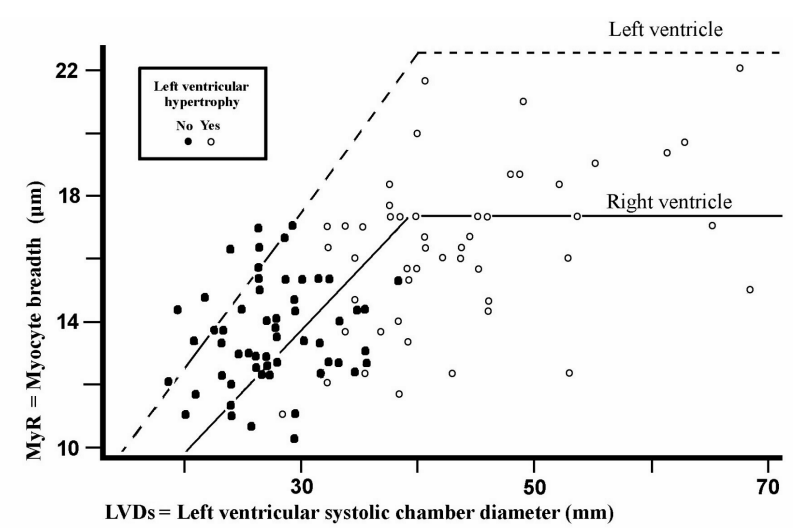

Figure 3: Mean myocyte breadth in RV versus systolic LV chamber diameter for specimens with or without LV hypertrophy. Upward sloping lines are radii from the graphic origin fit to the scatter of points by graphic inspection. The horizontal lines are regression fits through the scatter of points rightward of the $40 \mathrm{~mm}$ cutoff point using $\beta=0$, because $\beta$ does not differ from zero $(p>0.05)$. Dashed lines for LV are adapted from a previous publication

Photographic examples: Figure 4 illustrates what is typically encountered in the mid ranges of myocyte cross sectional breadth for normal or hypertrophied right ventricles (dots or circles respectively in Figure 3). For the right ventricle these photographic examples fall on the Figure 3 ordinate at MyR=13.5 $\mu \mathrm{m}$ and $17.6 \mu \mathrm{m}$. (Frames A and C in Figure 4).

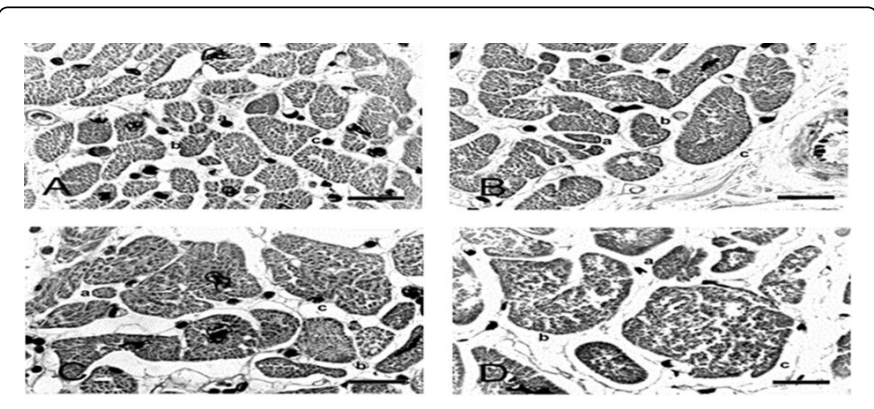

Figure 4: Average normal (A and $B$ ) and hypertrophied (C and D) right $(A$ and $C)$ and left (B and $D)$ ventricles are chosen to present high power fields that depict smallest, average, and largest ( $\mathrm{a}, \mathrm{b}$ and c) transverse dimensions in each frame $(\mathrm{H} \& \mathrm{E}$, bars $=20 \mathrm{~m})$.

Comparable examples for the left ventricle are also illustrated in Figure 4 Frames $\mathrm{B}$ and D (these fall on the Figure 3 ordinates $\mathrm{MyL}=14.7 \mu \mathrm{m}$ and $22.2 \mu \mathrm{m}$ ). Within each of these high power fields the letters $\mathrm{a}, \mathrm{b}$ and $\mathrm{c}$ identify the smallest, average, and largest breadths. These sizes were determined from the series of 60 measured profiles at each of the sites.
Midwall fractional shortening: The two variables, stroke volume and midwall circumferential excursion, are plotted in Figure 5 for pools of cases grouped by increasing midwall diastolic circumference. The ratio of excursion to circumference is fractional shortening (not plotted). The numerator of this ratio, excursion, reaches a peak around circumference of $200 \mathrm{~mm}$ and generally trends downward with further chamber dilatation. The trend is irregular because of few cases within the groups, especially those with the largest diameters. The widely used measurement, fractional shortening, displays associations with stroke volume that are seldom given consideration, and which call for some discussion.

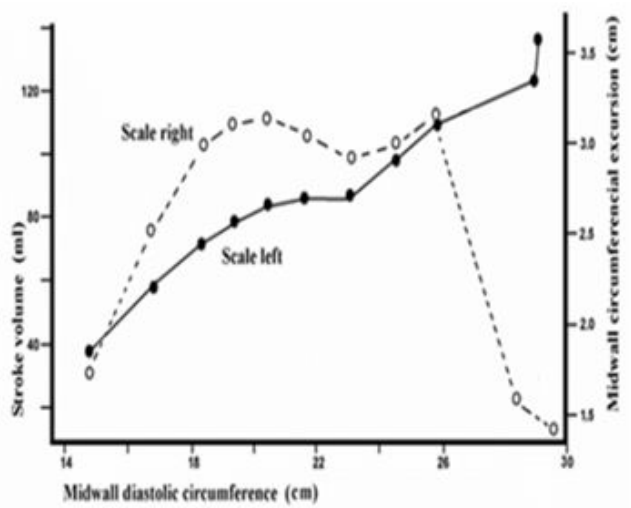

Figure 5: Echocardiographic data abstracted from published sources produced the plotted mean values for groupings within intervals of increasing diastolic chamber circumference.

\section{Discussion}

Reports of frequency distribution curves for LV myocyte breadths $[1,7,11,14]$ did not attempt statistical curve fitting, as done here for the first time. Those reported curves, however, often seemed consistent with absence of significant truncation as exemplified here in Figure 1. To explore this pattern more fully, curve fitting was done on each of the 104 cases. The rejection of fitted log normal curves by the ShapiroWilk test happened overly often in both right and left ventricles (Table 1) but did not favor the most hypertrophied specimens (Table 2).

The observed distributions were well enough described by the log normal form so that negative skewness could serve as a marker for truncation of the upper tails. Coexistence of negative skewness with myocyte hypertrophy is therefore an object of interest. Such coexistence occurred haphazardly across size groupings (Table 2), thereby suggesting the cautious conclusion that myocytes of all sizes undergo proportionately equal hypertrophy from infancy to adulthood and into pathological hypertrophy thereafter, i.e. allometric growth $[11,12]$. This outcome is inconsistent with the proposals that necrosis [15,16], apoptosis [2], or myocyte splitting [7] might be producing the limitations to hypertrophy observed here.

The systematic sampling of posterior and lateral walls in the LV equatorial plane did not identify either region as significantly different from the others in this series of cases. This observation has been noted before in some reports [1,11]. It therefore seems appropriate to propose that the significant heterogeneity found here for MyL between sites most likely occurs sporadically from one sample to another 
without regard to anatomic location. Findings for the right ventricle, MyR, reiterate these results. The unexpected finding of sporadic variation from place to place in myocyte mean dimensions therefore poses newly raised questions for testing at a later time. It should be noted that these variations are often visible in comparisons between adjacent high power fields under the microscope, not requiring large intervening distances.

Figure 3 shows that myocytes in the RV behave in some ways like those in the $\mathrm{LV}$ because both $\mathrm{MyR}$ and $\mathrm{MyL}$ attain the limit to transverse hypertrophy at or near LVDs $\approx 40 \mathrm{~mm}$. Yet the magnitude of the constant mean myocyte breadth beyond this limiting condition is conspicuously different between the ventricles (near $\mathrm{MyL}$ MyR=21.9 $\mu \mathrm{m}-17.5 \mu \mathrm{m}$ at all values for LVDs above the $40 \mathrm{~mm}$ cutoff level). Unlike the RV, the LV myocytes do not cease enlarging at $\mathrm{MyL}=17.5 \mu \mathrm{m}$; they unremittingly continue to hypertrophy further. Modeling of this difference does not require introducing variation in subcellular events such as contractile weakness, because it is well explained by the simple operation of geometry. The attained size of myocytes cannot be the influence that generates the $17.5 \mu \mathrm{m}$ limit for mean transverse dimension in the RV if their properties are similar in the two ventricles.

Some kind of threshold for both MyR and MyL appears to be situated around LVDs $\approx 40 \mathrm{~mm}$. Near this threshold the LV was previously found to undergo a gradual change in behavior [5,9]. Using heuristically the spherical model for LV dimensions, the systolic and diastolic chamber radii can be called Rs and Rd respectively. Hence the midwall fractional shortening, MFS, is roughly proportional to endocardial fractional shortening [17] as measured by MFS $\propto(\mathrm{Rd}-$ $\mathrm{Rs)} / \mathrm{Rd}$. Both $\mathrm{Rd}$ and Rs must increase to handle the rising volume overload of overweight and its accompanying enhanced stroke volume. Stroke volume can be set to $\mathrm{SV} \approx 4 \times \pi \times\left(\mathrm{Rd}^{3}-\mathrm{Rs}^{3}\right) / 3$ (prior to the Teichholz adjustment as used in this data set [18]). The numerator of MFS is a difference of linear terms while SV is a difference of those terms cubed; also the term Rd occupies the denominator only in MFS. Applying these principles to physiological conditions showed that enhanced chamber size in company with rising values for SV require progressively smaller changes in the midwall circumferential excursion, $2 \times \pi \times(\mathrm{Rd}-\mathrm{RS})$ (Figure 5, using echocardiographic data from published sources). Since this declining excursion is proportional to the numerator in MFS, the increasing Rd in the denominator forces fractional shortening (MFS (Rd - Rs)/Rd) to decline steeply, and this can be solely for geometrical reasons without need to invoke alterations in contractile strength.

Further consideration of these principles can be extended to the behavior of sarcomeres [5]. As the chamber dilates, the number of sarcomeres must increase so that the excursion per sarcomere progressively declines, as just reviewed. When approaching the limit to myocyte length, the sarcomere excursion will progress toward zero, as found in myofibers observed by noninvasive imaging $[19,20]$. The proposal that alterations in ventricular geometry might precede and cause contractile weakening has recently received some attention $[21,22]$. That proposal, along with some other alternative models, is expanded in detail elsewhere [5].

Limitations in this study are numerous and conspicuous, and most of them are often shared with other past and present efforts. Chief among these is the surprising difficulty in measuring the in situ dimensions of cardiomyocytes, as illustrated in Figure 4. Until a gold standard becomes available probes along these lines must remain tentative. Most needed are direct measurements of myocyte lengths as well as breadth rather than using only an estimate of the mean length. Also, the exclusion of ischemic, valvular, and specifically right ventricular disorders leaves serious gaps. Also needing confirmation or rejection is the suggestion, arising from this series of studies [8], of equal importance for lean and fat body mass in driving myocyte hypertrophy. Results described here are concerned with the behavior of the means of grouped cases while individual departures from these means remain to receive consideration. If hypertension can be viewed as a composite of two forms, neurohumoral and renal microischemic [23], then studies such as this one cannot distinguish which of these forms is most important for cardiac hypertrophy.

In conclusion, myocytes of all sizes throughout the right ventricle in each case appear to attain a limit to transverse dimensional hypertrophy simultaneously irrespective of myocyte size. This perspective is exemplified by the pool of most hypertrophied specimens in Figure 1, which illustrates the lack of truncation in the upper tail. The right ventricle reaches this postulated limit at average myocyte sizes somewhat smaller than those simultaneously observed in the left ventricle. The composite of the findings implies that the attained myocyte size does not, in itself, impose the limit upon the largest cells. Rather, what emerges is that a fixed number of myocytes composing a ventricle must each grow larger in accordance with the LV spherical model irrespective of contractile strength of individual myocytes. The geometry calls for midwall fractional shortening to taper off when delivering more stroke volume and rising stroke work with increasing body weight; this tapering approaches zero at the final limit to hypertrophy. This result argues against energy starvation [16] or other subcellular events to explain the limitation of myocyte hypertrophy. Cardiac disorders are among the natural conditions most frequently encountered by forensic pathologists; and this circumstance puts them on the front line to pursue these matters. It would be of some value if this report can promote ongoing discussions.

\section{References}

1. Campbell SE, Korecky B, Rakusan K (1991) Remodeling of myocyte dimensions in hypertrophic and atrophic rat hearts. Circ Res 68: 984-996.

2. Onodera T, Tamura T, Said S, McCune SA, Gerdes AM (1998) Maladaptive remodeling of cardiac myocyte shape begins long before failure in hypertension. Hypertension 32: 753-757.

3. Kerckhoffs RC, Omens J, McCulloch AD (2012) A single strain-based growth law predicts concentric and eccentric cardiac growth during pressure and volume overload. Mech Res Commun 42: 40-50.

4. Messerli FH, Sundgaard-Riise K, Reisen E, Dreslinski G, Dunn FG, et al. (1983) Disparate cardiovascular effects of obesity and arterial hypertension. Am J Med 74: 808-812.

5. Tracy RE (2016) Heart failure and limit to cardiomyocyte hypertrophy: Lessons from autopsies. Eur J Forensic Sci 3: 29-38.

6. Gerdes AM (2002) Cardiac myocyte remodeling in hypertrophy and progression to failure. J Card Fail 8: S264-S268.

7. Linzbach J (1960) Heart failure from the point of view of quantitative anatomy. Am J Cardiol 5: 370-382.

8. Tracy RE, Sander GE (2011) Histologically measured cardiomyocyte hypertrophy correlates with body height as strongly as with body mass index. Cardiol Res Pract 2011: 658958.

9. Tracy RE (2012) Cardiomyocyte size estimated from noninvasive measurements of left ventricular wall thickness and chamber diameter. J Am Soc Hypertens 6: 185-192.

10. de Simone G, Devereux RB, Roman MJ, Ganau A, Saba PS, et al. (1994) Assessment of left ventricular function by the mid wall fractional shortening/end-systolic stress relation in human hypertension. J Am Coll Cardiol 23: 1444-1451. 
Citation: Tracy RE (2016) Limit to Cardiomyocyte Hypertrophy in Right and Left Ventricles: Possible Precursors of Systolic Heart Failure. J Forensic Res 7: 345. doi:10.4172/2157-7145.1000345

Page 7 of 7

11. Korecky B, Rakusan K (1978) Normal and hypertrophic growth of the rat heart: changes in cell dimensions and number. Am J Physiol 234: H123H128.

12. de Simone G, Devereux RB, Kimball TR, Mureddu GF, Roman MJ, et al. (1998) Interaction between body size and cardiac workload: Influence on left ventricular mass during body growth and adulthood. Hypertension 31: 1077-1082.

13. Barry WH (1998) Load-dependent myocyte dysfunction. Circulation 97: 2297-2298.

14. Katz AM (1990) Cardiomyopathy of overload: A major determinant of prognosis in congestive heart failure. N Engl J Med 322: 100-110.

15. Karsner HT, Saphir O, Todd TW (1925) The State of the cardiac muscle in hypertrophy and atrophy. Am J Pathol 1: 351-371.

16. Grossman W, Jones E, McLaurin LP (1975) Wall stress and patterns of hypertrophy in the human left ventricle. J Clin Invest 56: 56-64.

17. Teichholz LE, Kreulen T, Herman MV, Gorlin R (1976) Problems in echocardiographic volume determinations: echocardiographic- angiographic correlations in the presence or absence of asynergy. Am J Cardiol 37: 7-11.

18. Sonnenblinck EH, Ross J Jr, Covell JW, Spotnitz HM, Spiro D (1967) The ultrastructure of the heart in systole and diastole. Changes in sarcomere length. Circ Res 21: 423-431.

19. MacGowan GA, Shapiro EP, Azhari H, Siu CO, Hees PS, et al. (1997) Noninvasive measurement of shortening in the fiber and cross-fiber directions in the normal human left ventricle and in idiopathic dilated cardiomyopathy. Circulation 96: 535-541.

20. Dipla K, Mattiello JA, Jeevanandam V, Houser SR, Margulies KB (1998) Myocyte recovery after mechanical circulatory support in humans with end-stage heart failure. Circulation 97: 2316-2322.

21. Houser SR, Margulies KB (2003) Is depressed myocyte contractility centrally involved in heart failure? Circ Res 92: 350-358.

22. Tracy RE (2011) Renal vasculature in essential hypertension: A review of some contrarian evidence. Contrib Nephrol 169: 327-336. 